\title{
AERODYNAMICS OF AN UNSTEADY PARTICLE FLOW IN THE CHUTE
}

\author{
O. A. Averkova1, I. N. Logachev', and K. I. Logachev', \\ ${ }^{1}$ Belgorod State Technological University named after V.G. Shukhov, \\ 308012 Belgorod, Russia, Kostukova str., 46 \\ e-mail: $\{$ logachevin, olga_19572004, kilogachev\}@mail.ru
}

Keywords: Granular Materials, Bulk Material Transfer, Air Suction, Local Exhaust Ventilation.

\begin{abstract}
The conducted studies are aimed at improving the theoretical basis for design of ventilated suction hoods of bulk material transfer groups, i.e. the most typical and common sources of dust emissions in reprocessing of dust-forming materials.Unsteady processes occur in the chute at the equipment start-up or short-time loading of bulk material. Assess the force action exerted by the flow on air for two cases: at an instantaneous change and a gradual one (smooth) in the material flow. 'It was theoretically demonstrated that at instant chuting of a heated bulk material an increase in the induction pressure is strongly related with a variation in the transferred material flow, and due to a considerable inertness of the intercomponent heat exchange temperature variations are far behind of variations in the transferred material quantity. A gradual variation in the flow rate of particles at the beginning and at the end of a material transfer is associated with excessive (peak) pressures against steady-state values. This is explained by the maximum induction head at a certain material flow rate. There are no pressure surges if the material flow rate does not reach the necessary value.
\end{abstract}




\section{INTRODUCTION}

The conducted studies are aimed at improving the theoretical basis for design of ventilated suction hoods of bulk material transfer groups, i.e. the most typical and common sources of dust emissions in reprocessing of dust-forming materials [1-8, 12-14].

Unsteady processes occur in the chute at the equipment start-up or short-time loading of bulk material. Assess the force action exerted by the flow on air for two cases: at an instantaneous change and a gradual one (smooth) in the material flow.

\section{SUDDEN CHANGE IN THE MATERIAL FLOW}

Examine the change in the forces of the induction and thermal pressures and the starting and stop times of the material feeding.

Observe the change in the induction pressure by the example of the pressure distribution along the length of the vertical pipe in case of its irregular load with bulk material of moderate temperature, thereby preventing from the heat and mass exchange. Imagine that the lower end of the pipe is closed to the air passage, i.e. $v_{2}=0$, but open to the passage of material.

With these simplifications [1]

$$
\frac{\partial v_{2}}{\partial t}=-\frac{\partial P}{\partial x} \cdot \frac{1}{\rho_{2}}+\kappa_{m} \beta_{1} \psi \frac{v_{1}^{2}}{2} .
$$

Examine a "hard" loading of material (instant inlet of material). Then, at an arbitrary point of time $t>0$, the change in the material flow along the length will be of a step-type shape.

$$
\begin{gathered}
G_{1}=G_{\max } \text { at } x<x_{0}, \\
G_{1}=0 \text { at } x>x_{0} .
\end{gathered}
$$

The kink will be moved down. Let us assume that its movement speed is

$$
\bar{v}_{1}=\frac{1}{l} \int_{0}^{l} v_{1} d x \approx 0,5\left(v_{1 \mathrm{H}}+v_{1 k}\right) .
$$

The analytical expression of the change in the material flow is as follows

$$
G_{1}=G_{\max } \cdot f\left(x-v_{1} t\right),
$$

where

$$
\begin{aligned}
& f\left(x-v_{1} t\right)=0 \text { at } x-v_{1} t>0 ; \\
& f\left(x-v_{1} t\right)=1 \text { at } x-v_{1} t<0 .
\end{aligned}
$$

For simplicity of notation, the symbol of averaging (a line above $v_{1}$ ) is omitted here and hereinafter.

Express the function $f\left(x-v_{1} t\right)$ by means of the infinite Fourier series [9]

$$
f\left(x-v_{1} t\right)=\frac{1}{2}+\sum_{n=1}^{\infty} \frac{\cos \pi n-1}{n \pi} \sin \frac{\pi}{l_{\infty}}\left(x-v_{1} t\right),
$$

where

$$
l_{\infty}=\lim _{t \rightarrow \infty}\left(x-v_{1} t\right) .
$$


Then, the volume concentration of material is

$$
\beta_{1}=\beta_{1 \infty}\left[\frac{1}{2}+\sum_{n=1}^{\infty} \frac{\cos n \pi-1}{n \pi} \sin n \frac{\pi}{l_{\infty}}\left(x-v_{1} t\right)\right]
$$

where

$$
\beta_{1 \infty}=\frac{G_{1 \max }}{\rho_{1} S_{\varkappa v_{1}} v_{1}}
$$

or, solving the sine function of difference of two angles,

$$
\left.\beta_{1}=\beta_{1 \infty}\left[\frac{1}{2}+\sum_{n=1}^{\infty} \frac{\cos n \pi-1}{n \pi} \sin n \frac{\pi}{l_{\infty}} x \cos n \frac{\pi}{l_{\infty}} t v_{1}-\sum_{n=1}^{\infty} \frac{\cos n \pi-1}{n \pi} \cos n \frac{\pi}{l_{\infty}} x \sin n \frac{\pi}{l_{\infty}} v_{1} t\right)\right]
$$

Write mass transfer equation [1]

$$
\frac{\partial \rho_{2}}{\partial t}+\frac{\partial \rho_{2} v_{2}}{\partial x}=0
$$

Assuming that that process is adiabatic (with factor $\chi$ )

$$
\frac{P}{P_{0}}=\left(\frac{\rho_{2}}{\rho_{0}}\right)^{\chi}
$$

simple transformations made, taking into account that the density of the medium does not change significantly, equality (10) can be reduced to

$$
\frac{\partial v_{2}}{\partial x}=-\frac{\rho_{0}}{\bar{\rho}_{2} P_{0} \chi} \frac{\partial P}{\partial t}
$$

where $P_{0}, \rho_{0}$ are the pressure and density of air in the pipe before inputting the material.

Taking account of correlation (12), after differentiation of equality (1) with respect to $x$, we obtain an inhomogeneous equation of acoustics:

$$
\frac{\partial^{2} P}{\partial t^{2}}=v_{a}^{2} \frac{\partial^{2} P}{\partial x^{2}}-v_{a}^{2} \frac{\partial}{\partial x} \kappa_{m} \beta_{1} \psi \frac{v_{1}^{2}}{2} \bar{\rho}_{2},
$$

where $v_{a}=\sqrt{\chi P_{0} / \rho_{0}}$ is the propagation speed of elastic disturbances (speed of sound), $\mathrm{m} / \mathrm{sec}$.

Assume that the force of the dynamic interaction is constant

$$
\kappa_{m} \psi \frac{v_{1}^{2}}{2} \bar{\rho}_{2}=\text { const } .
$$

Under this assumption, a linear pressure distribution along the pipe would exist for stationary conditions

$$
P_{c m}=P_{0}+\kappa_{m} \beta_{1 \infty} \psi \frac{v_{1}^{2}}{2} \bar{\rho}_{2} x
$$

or

$$
P_{\infty}=P_{l} \frac{x}{l},
$$


where $P_{l}$ is the excessive pressure at the end of the pipe

$$
P_{l}=\kappa_{m} \beta_{10} \psi \frac{v_{1}^{2}}{2} \bar{\rho}_{2} l
$$

$P_{c m}, P_{\infty}$ are the absolute and excessive pressures, respectively, in a stable process, $\mathrm{Pa}$.

Taking into account the assumptions made and designations, the last term of the righthand side of equation (13) can be written as

$$
\begin{aligned}
v_{a}^{2} \frac{\partial}{\partial x} \kappa_{m} \beta_{1} \psi & \frac{v_{1}^{2}}{2} \bar{\rho}_{2}=v_{a}^{2} \frac{P_{l}}{l l_{\infty}}\left[\sum_{n=1}^{\infty}(\cos n \pi-1) \cdot \cos n \pi \frac{x}{l_{\infty}} \cos n \pi \frac{v_{1} t}{l_{\infty}}+\right. \\
& \left.+\sum_{n=1}^{\infty}(\cos n \pi-1) \sin n \pi \frac{x}{l_{\infty}} x \cdot \sin n \pi \frac{v_{1} t}{l_{\infty}}\right]
\end{aligned}
$$

Formulate the initial and boundary conditions. Pressure $P$ in equation (13) is to mean the excessive pressure. As dead air was in the pipe before the input of material, and the absolute pressure in it was $P_{0}$ :

$$
\begin{aligned}
& \left.P\right|_{t=0}=0 ; \\
& \left.v_{2}\right|_{t=0}=0 .
\end{aligned}
$$

Integrate equation (12) to obtain

$$
v_{2}=\left.v_{2}\right|_{x=0}-\frac{\rho_{0}}{P_{0} \chi} \int_{0}^{x} \frac{\partial P}{\partial t} d x
$$

where from, taking into account (20), obtain the second initial condition

$$
\left.\frac{\partial P}{\partial t}\right|_{t=0}=0 \text {. }
$$

The boundary condition for the open end of the pipe (input) will be

$$
P(0, t)=0 \text {. }
$$

For the lower end, taking in account the air tightness of the pipe bottom,

$$
v_{2}(l, t)=0 \text {. }
$$

Integrate the initial equation (1) to obtain

$$
v_{2}=v_{2}(x, 0)+\int_{0}^{t}\left(-\frac{\partial P}{\partial x} \cdot \frac{1}{\rho_{2}}+\kappa_{m} \beta_{1} \psi \frac{v_{1}^{2}}{2}\right) d t .
$$

Taking into account (24), obtain the second boundary condition:

$$
\left.\frac{\partial P}{\partial x}\right|_{x=l}=\left.\kappa_{m} \beta_{1} \psi \bar{\rho}_{2} \frac{v_{1}^{2}}{2}\right|_{x=l},
$$

which can be written as follows, having in mind correlation (7), after some transformations

$$
\left.P\right|_{x=l}=2 \frac{P_{l}}{l} \sum_{n=1}^{\infty} \frac{1-\cos n \pi}{n \pi} \cos ^{2} n \pi \frac{l}{2 l_{\infty}} \sin n \pi \frac{v_{1} t}{l_{\infty}}+\frac{P_{l}}{l} \sum_{n=1}^{\infty} \frac{\cos n \pi-1}{n \pi} \sin n \pi \frac{l}{l_{\infty}} \cos n \pi \frac{v_{1} t}{l_{\infty}} .
$$


Thus, we have to solve the following inhomogeneous differential partial equation with second-order partial derivatives [10] with the initial conditions (19) and (22) and the boundary conditions (23) and (27)

$$
\frac{\partial^{2} P}{\partial t^{2}}=v_{a}^{2} \frac{\partial P}{\partial x^{2}}-v_{a}^{2} \frac{P_{l}}{l l_{\infty}} \sum_{n=1}^{\infty}(\cos n \pi-1) \cos n \pi \frac{x}{l_{\infty}} \cos n \pi \frac{v_{1} t}{l_{\infty}}-v_{a}^{2} \frac{P_{l}}{l l_{\infty}} \sum_{n=1}^{\infty}(\cos n \pi-1) \sin n \pi \frac{x}{l_{\infty}} \sin n \pi \frac{v_{1} t}{l_{\infty}}
$$

The solution will be calculated as a sum of functions

$$
P=u+\xi,
$$

where $u$ is the solution of equation (28) only with the boundary conditions; $\xi$ is the solution of this equation without a constant term with the following initial and boundary conditions:

$$
\begin{aligned}
& \left.\xi\right|_{t=0}=-\left.u\right|_{t=0} \\
& \left.\frac{\partial \xi}{\partial t}\right|_{t=0}=-\left.\frac{\partial u}{\partial t}\right|_{t=0} \\
& \left.\xi\right|_{x=0}=0 \\
& \left.\frac{\partial \xi}{\partial x}\right|_{x=l}=0
\end{aligned}
$$

The task to determine $u$ can, in turn, be divided into two sub-tasks:

a) the solution of the equation

$$
\frac{\partial^{2} u}{\partial t^{2}}=v_{a}^{2} \frac{\partial^{2} u}{\partial x^{2}}-v_{a}^{2} \frac{P_{l}}{l \cdot l_{\infty}} \sum_{n=1}^{\infty}(\cos n \pi-1) \cos n \pi \frac{x}{l_{\infty}} \cos n \pi \frac{v_{1} t}{l_{\infty}}
$$

with

$$
\left.u\right|_{x=0}=0 ;\left.\quad \frac{\partial u}{\partial x}\right|_{x=l}=\frac{P_{l}}{l} \sum_{n=1}^{\infty} \frac{\cos n \pi-1}{n \pi} \sin n \pi \frac{l}{l_{\infty}} \cos n \pi \frac{v_{1} t}{l_{\infty}} ;
$$

b) the solution of the equation

$$
\frac{\partial^{2} u}{\partial t^{2}}=v_{a}^{2} \frac{\partial^{2} u}{\partial x^{2}}-v_{a}^{2} \frac{P_{l}}{l \cdot l_{\infty}} \sum_{n=1}^{\infty}(\cos n \pi-1) \sin n \pi \frac{x}{l_{\infty}} \sin n \pi \frac{v_{1} t}{l_{\infty}}
$$

with

$$
\left.u\right|_{x=0}=0 \quad ;\left.\quad \frac{\partial u}{\partial x}\right|_{x=l}=\frac{P_{l}}{l} \sum_{n=1}^{\infty} \frac{\cos n \pi-1}{n \pi}\left(1+\cos n \pi \frac{l}{l_{\infty}}\right) \sin n \pi \frac{v_{1} t}{l_{\infty}} .
$$

Subtask a). Try the solution of equation (36) as follows

$$
u_{a}=\sum_{n=1}^{\infty} X_{n}(x) \cos n \pi \frac{v_{1} t}{l_{\infty}} .
$$

Inserting this solution into the initial equation after obvious reductions, we obtain

$$
\frac{d^{2} X_{n}(x)}{d t^{2}}+X_{n}(x) \cdot\left(\frac{n \pi M_{v}}{l_{\infty}}\right)^{2}=\frac{P_{l}}{l \cdot l_{\infty}}(\cos n \pi-1) \cdot \cos n \pi \frac{x}{l_{\infty}},
$$

where 


$$
M_{v}=v_{1} / v_{a}
$$

Integrate this equation, with

$$
\begin{gathered}
X_{n}(0)=0, \\
\left.\frac{d X_{n}(x)}{d x}\right|_{x=l}=\frac{P_{l}}{l} \frac{\cos n \pi-1}{n \pi} \cdot \sin n \pi \frac{l}{l_{\infty}}
\end{gathered}
$$

based on the conditions (35) we obtain

$$
X_{n}(x)=\Lambda\left[\Phi \sin \pi n \frac{x}{l_{\infty}} M_{v}-\cos n \pi \frac{x}{l_{\infty}} M_{v}+\cos n \pi \frac{x}{l_{\infty}}\right]
$$

and the solution of Subtask a) is

$$
u_{a}=\sum_{n=1}^{\infty} \Lambda\left[\Phi \sin \pi n \frac{x}{l_{\infty}} M_{v}-\cos n \pi \frac{x}{l_{\infty}} M_{v}+\cos n \pi \frac{x}{l_{\infty}}\right] \cdot \cos \pi n \frac{v_{1} t}{l_{\infty}},
$$

where for simplicity of notation we assume

$$
\begin{gathered}
\Lambda=\frac{P_{l} \cdot l_{\infty}}{(n \pi)^{2} l} \cdot \frac{\cos n \pi-1}{M_{v}^{2}-1}, \\
\Phi=\left(M_{v} \sin \pi n \frac{l}{l_{\infty}}-\sin \pi n \frac{l}{l_{\infty}} M_{v}\right) / \cos n \pi \frac{l}{l_{\infty}} M_{v} .
\end{gathered}
$$

Subtask b). Equation (36) is solved in the same way.

Obtain

$$
u_{\delta}=\sum_{n=1}^{\infty} \Lambda\left(\sin \pi n \frac{x}{l_{\infty}}-\Omega \sin \pi n \frac{x}{l_{\infty}} M_{v}\right) \cdot \sin \pi n \frac{v_{1} t}{l_{\infty}}
$$

where

$$
\Omega=\frac{M_{v}^{2}-1}{M_{v}} \cdot \frac{1+\cos n \pi \frac{l}{l_{\infty}}}{\cos n \pi \frac{l}{l_{\infty}} M_{v}}+\frac{1}{M_{v}} .
$$

We now calculate the function $\xi$, which is the solution of equation

$$
\frac{\partial^{2} \xi}{\partial t^{2}}=v_{a}^{2} \frac{\partial^{2} \xi}{\partial x^{2}}
$$

with the boundary conditions (32) and (33) and with the following initial conditions based on equalities (43) and (46)

$$
\begin{aligned}
& \left.\xi\right|_{t=0}=-\left.\left(u_{a}+u_{\delta}\right)\right|_{t=o}=-\sum_{n=1}^{\infty} \Lambda\left[\Phi_{n} \sin \pi n \frac{x}{l_{\infty}} M_{v}-\cos n \pi \frac{x}{l_{\infty}} M_{v}+\cos n \pi \frac{x}{l_{\infty}}\right]=f(x) ; \\
& \left.\frac{\partial \xi}{\partial t}\right|_{t=0}=-\left.\left(\frac{\partial u_{a}}{\partial t}+\frac{\partial u_{\delta}}{\partial t}\right)\right|_{t=0}=-\sum_{n=1}^{\infty} \Lambda n \pi \frac{v_{1}}{l_{\infty}}\left(\sin \pi n \frac{x}{l_{\infty}}-\Omega \sin \pi n \frac{x}{l_{\infty}} M_{v}\right)=F(x) .
\end{aligned}
$$


We solve it using the Fourier method - by expansion of the function in series in the orthogonal function system $\sin \frac{2 n+1}{2 l} \pi x[11]$.

The solution is

$$
\xi=\sum_{n=0}^{\infty}\left[a_{\kappa} \cos \frac{2 n+1}{2 l} \pi v_{a} t+\delta_{\kappa} \sin \frac{2 n+1}{2 l} \pi v_{a} t\right] \sin \frac{2 n+1}{2 l} \pi x,
$$

where

$$
\begin{aligned}
& a_{\kappa}=\frac{2}{l} \int_{0}^{l} f(x) \sin \frac{2 n+1}{2 l} \pi x d x ; \\
& \delta_{\kappa}=\frac{4}{(2 n+1) \pi v_{a}} \int_{0}^{l} F(x) \sin \frac{2 n+1}{2 l} \pi x d x .
\end{aligned}
$$

Inserting into equality (29) the obtained functions $\xi, u_{a}, u_{\delta}$, we obtain the desired solution. For a small falling velocity of the material $\left(v_{1} / v_{2}<<1\right)$, the solution can be reduced to a simpler form after a number of simplifications

$$
P=P_{l} \frac{l_{\infty}}{l} \sum_{n=1}^{\infty} \frac{1-\cos n \pi}{(n \pi)^{2}} \cos n \pi \frac{x-v_{1} t}{l_{\infty}}+\frac{1}{2} P_{l} \frac{l_{\infty}}{l}\left(\frac{v_{1} t}{l_{\infty}}-\frac{1}{2}\right)+\frac{1}{2} P_{l} \frac{x}{l} .
$$

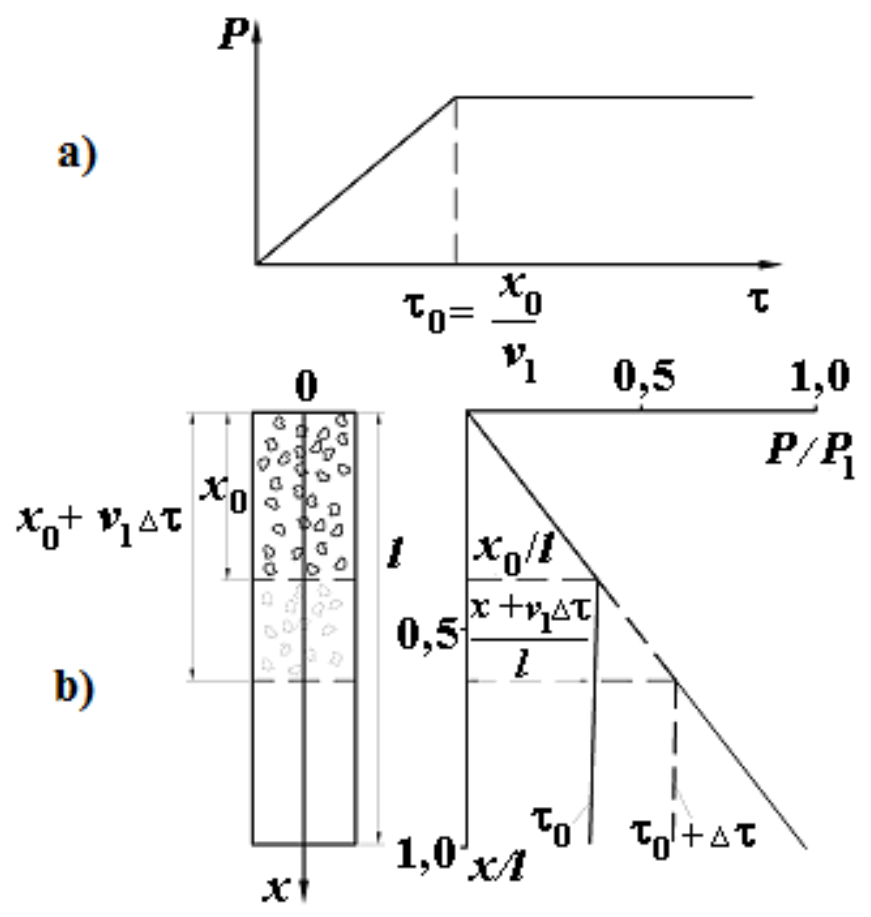

Figure 1: Change in the induction pressure over time (a) and along the tube (b) in case of an instant loading of bulk material

As can be seen from the graph (Figure 1) plotted based on this equation, the pressure in an arbitrary section $x_{0}$ increases in this section up to the maximum according to $t_{0} \equiv \tau_{0}=x_{0} / v_{1}$ s, i.d., as soon as the first particles of the material reach the section under consideration.

Along the entire pipe length, the pressure reaches its maximum value as soon as the pipe is filled with the falling material. 
Thus, a change in the induction pressure is "rigidly" connected with the change in the material flow. The steady mode of dynamic interaction between the material and the air occurs

almost simultaneously with a constant flow of material in all sections of the pipe.

In contrast to the dynamic interaction, temperature changes "fall behind" the fluctuations significantly in the mode of the material transfer.

To see this, consider the same task having slightly simplified it. Assume that the air thermal conductivity is high supposing that the same temperature is instantly set in all the sections of the pipe. Thus, the temperature will depend on time only. With the previous assumptions, the heat exchange equation can be as follows

$$
\frac{d}{d \tau} \beta_{2} \rho_{2} c_{2} t_{2}=\alpha \kappa_{s} \beta_{1 l}\left(t_{1}-t_{2}\right)-4 \frac{k}{D}\left(t_{2}-t_{0}\right),
$$

where $k$ is a coefficient of heat exchange with surrounding air, $\mathrm{BT} /\left(\mathrm{m}^{2} \cdot{ }^{\circ} \mathrm{K}\right) ; \beta_{l l}$ is the volume concentration of material in the chute.

Taking into account the step-type change in the flow rate, a solution is obtained for two intervals with $0<\tau<\frac{l}{v_{1}}$, with $\tau>\frac{l}{v_{1}}$ :

in the first interval

$$
\beta_{1 l}=\frac{G_{1} \tau}{\rho_{1} S_{\varkappa l} l}
$$

in the second interval

$$
\beta_{1 l}=\frac{G_{1}}{\rho_{1} S_{\varkappa} v_{1}} .
$$

Integrate equality (55) with the initial condition

$$
t_{2}(x, 0)=t_{0}
$$

to obtain:

a) with $0<\tau<\frac{l}{v_{1}}$

$$
t_{2}=t_{1}-\left(t_{1}-t_{0}\right)\left\{\exp \left[-\left(5 \tau+\frac{5 \tau^{2}}{2}\right)\right]+\frac{5}{\sqrt{B}} \exp \left(-\frac{y}{2}\right) \int_{B / \sqrt{B}}^{y} \operatorname{erf}\left(\frac{z^{2}}{2}\right) d z\right\}
$$

b) with $\tau>l / v_{1}$

$$
t_{2}=t_{2 \infty}-\left(t_{2 \infty}-t_{2 \mathrm{H}}\right) \exp \left[-\left(B \frac{l}{v_{1}}-B\right)\left(\tau-\frac{l}{v_{1}}\right)\right],
$$

where $t_{2 \mathrm{H}}$ is the air temperature in the pipe with $\tau=l / v_{1}$ is defined by equality (59); $t_{2 \infty}$ is the temperature in the pipe with $\tau \rightarrow \infty$

$$
t_{2 \infty}=\frac{B \frac{l}{v_{1}} t_{1}+5 t_{0}}{B \frac{l}{v_{1}}+5}
$$


$B, E$ are parameters introduced for simplicity of notation and equal to

$$
\begin{gathered}
B=\alpha \kappa_{S} G_{1} /\left(\rho_{2} c_{2} \rho_{1} S_{\varkappa} l\right) ; \\
B=4 \kappa /\left(D \rho_{2} c_{2}\right) ; \\
y=\sqrt{B} \tau+B / \sqrt{B} .
\end{gathered}
$$

For a heat sealed pipe $(E=0)$ we have:

a) with $0<\tau<l / v_{1}$

$$
t_{2}=t_{1}-\left(t_{1}-t_{0}\right) \exp \left(-B \tau^{2} / 2\right)
$$

b) with $\tau>l / v_{1}$

$$
t_{2}=t_{1}-\left(t_{1}-t_{0}\right) \exp \left[-B \frac{l}{v_{1}}\left(\tau-\frac{l}{2 v_{1}}\right)\right] .
$$

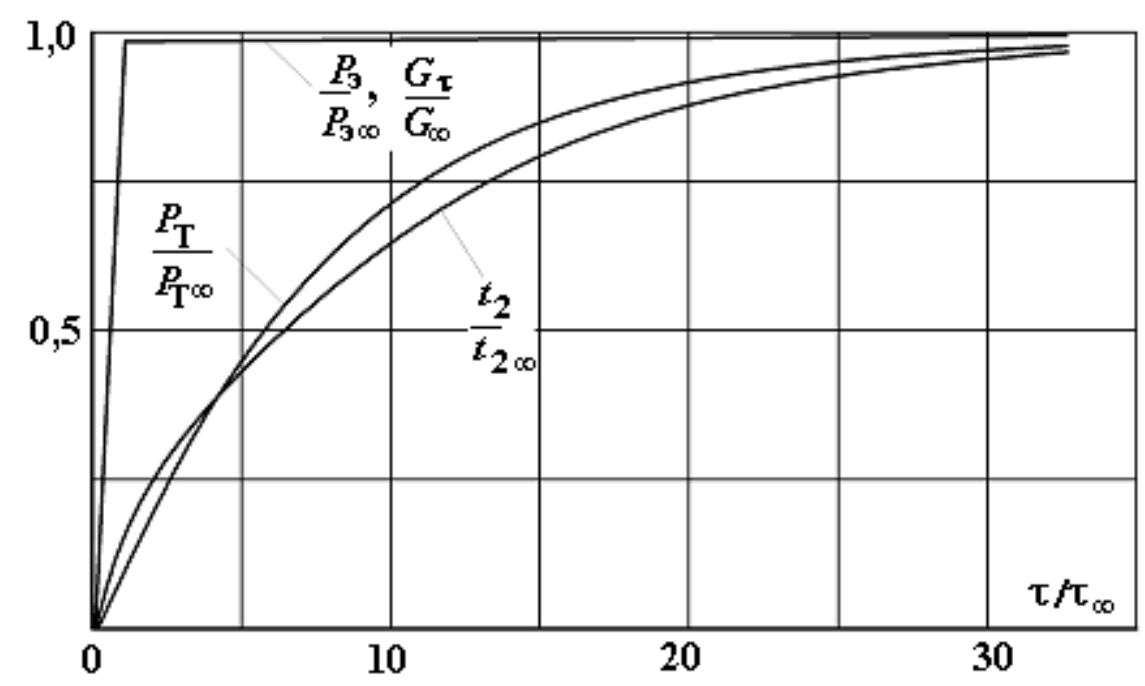

Figure 2: Temporal variation in temperature, thermal and induction pressures, mass of particles in the chute $\left(P_{\ni \infty}\right.$, $P_{T \infty}$ are the induction and thermal pressure with $\tau \rightarrow \infty ; G \tau, G \infty$ are the masses of particles in the chute at the moments in time $\tau$ and $\tau_{\infty}=l / \bar{v}_{1}$ )

Figure 2 shows the temperature curves plotted according to these formulas. It also shows the change in the thermal and induction pressures. As the curves show, the thermal pressure has a considerable "inertia" if compared with the induction one.

\section{SMOOTH CHANGE IN THE MATERIAL FLOW}

Given that the induction pressure is "rigidly" connected with the material flow, changes in the dynamic interaction can be assessed in the conditions of a changing flow of particles by means of the correlations obtained in the study of stationary flows. We can be used for a pressure at the end of the pipe, the lower end of which is closed to the air passage [1]:

$$
P_{\ni}=\psi_{0} \kappa_{m} \beta e^{\frac{-1,8 \sqrt{\beta \cdot 10^{3}}}{d_{\ni} \cdot 10^{3}}} \cdot l \frac{v_{1 k}^{2}}{2} \rho_{2} \frac{1-n^{3}}{3(1-n)},
$$


where $\beta$ is an averaged volume concentration of particles in the chute, which varies over time due to changes in flow.

As seen from equation (65), the induction pressure has a maximum at the volume concentration $\left(\beta_{\max }\right)$ defined by the equality

$$
2-\frac{1,8}{d_{\ni} \cdot 10^{3}} \sqrt{\beta_{\max } \cdot 10^{3}}=0 .
$$

Thus, if it appears that the concentration of particles varies widely from 0 to $\beta_{\infty}>\beta_{\max }$, pressure surges occur during an unsteady-state process. This is clearly seen in the curves in Figure 3. Here is a case of a bulk material transfer where its flow rate varies from 0 to the steady-state (constant) value $G_{1 \infty}$, then a stationary process $\left(\mathrm{G}_{1}=G_{1 \infty}\right)$ continues for a while and, finally, the flow rate decreases from $G_{1 \infty}$ down to 0 .
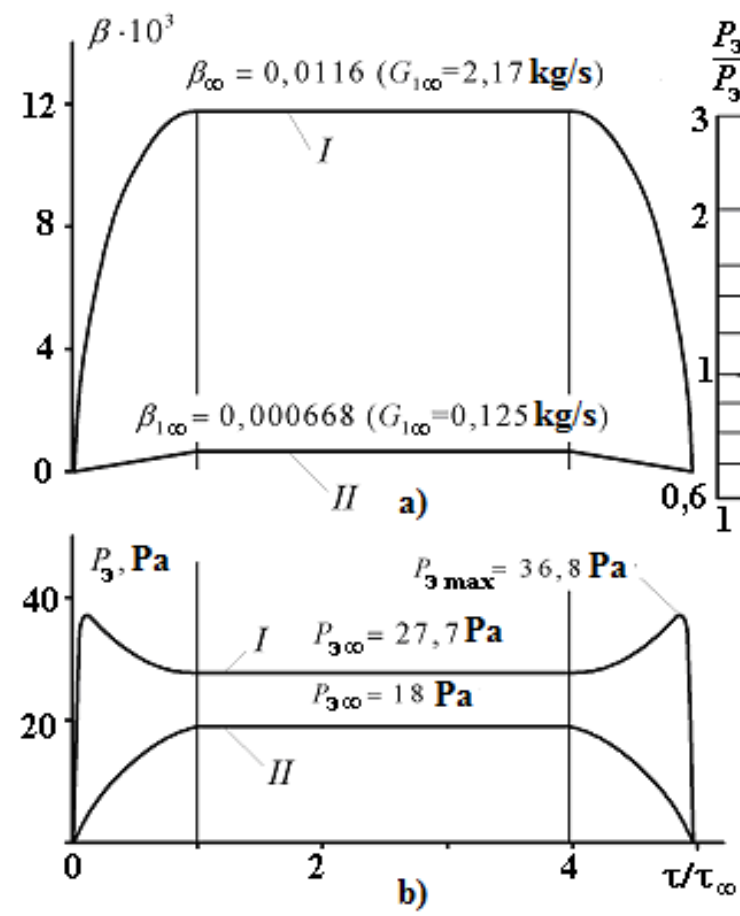

\section{$\underline{P_{3 \max }}$}

Figure 3: Change in the volume concentration and the induction pressure in the chute with a slow change in the material flow ( $\Delta$ is experimental data for conditions of granite bulking $d_{e}=1.88 \mathrm{~mm}$ in the chute at $\Theta=75^{\circ}, H=$

$$
3.3 \mathrm{~m}, S_{c h}=0.0169 \mathrm{~m}^{2} \text { ) }
$$

However, the pressure surge may be absent where the steady-state flow is so small that the volume concentration of particles in the chute is $\beta_{\infty}<\beta_{\max }$.

The maximum value of the induction pressure according to (67) is

$$
P_{\Im_{\max }}=\psi_{0} \kappa_{m} 10^{-3}\left(\frac{2 \cdot d_{\ni} \cdot 10^{3}}{1,8}\right)^{2} e^{-2} \cdot l \frac{v_{1 k}^{2}}{2} \rho_{2} \frac{1-n^{3}}{3(1-n)}
$$

or the relative value of the pressure surge is

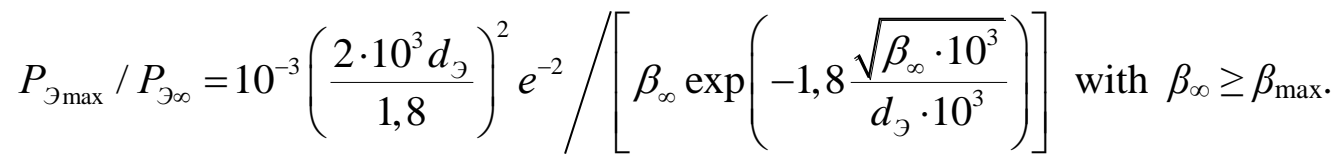


In studies of the induction properties of a bulk material flow in inclined chutes at the unit (Figure 4), a pressure surge was often observed when the material feeding from the upper bin both began and stopped. The value of this surge was significant at large material flows. No pressure rise was observed with small flows. A pressure rise is absolutely in line with equations (69) not only in qualitative terms but also in quantitative terms (see Figure 3.c). The observed falling of the thermal pressure behind the induction one, as well as the surge of the induction pressure during the start or stop of the process equipment must be taken into account when calculating the required volumes of aspirated air.

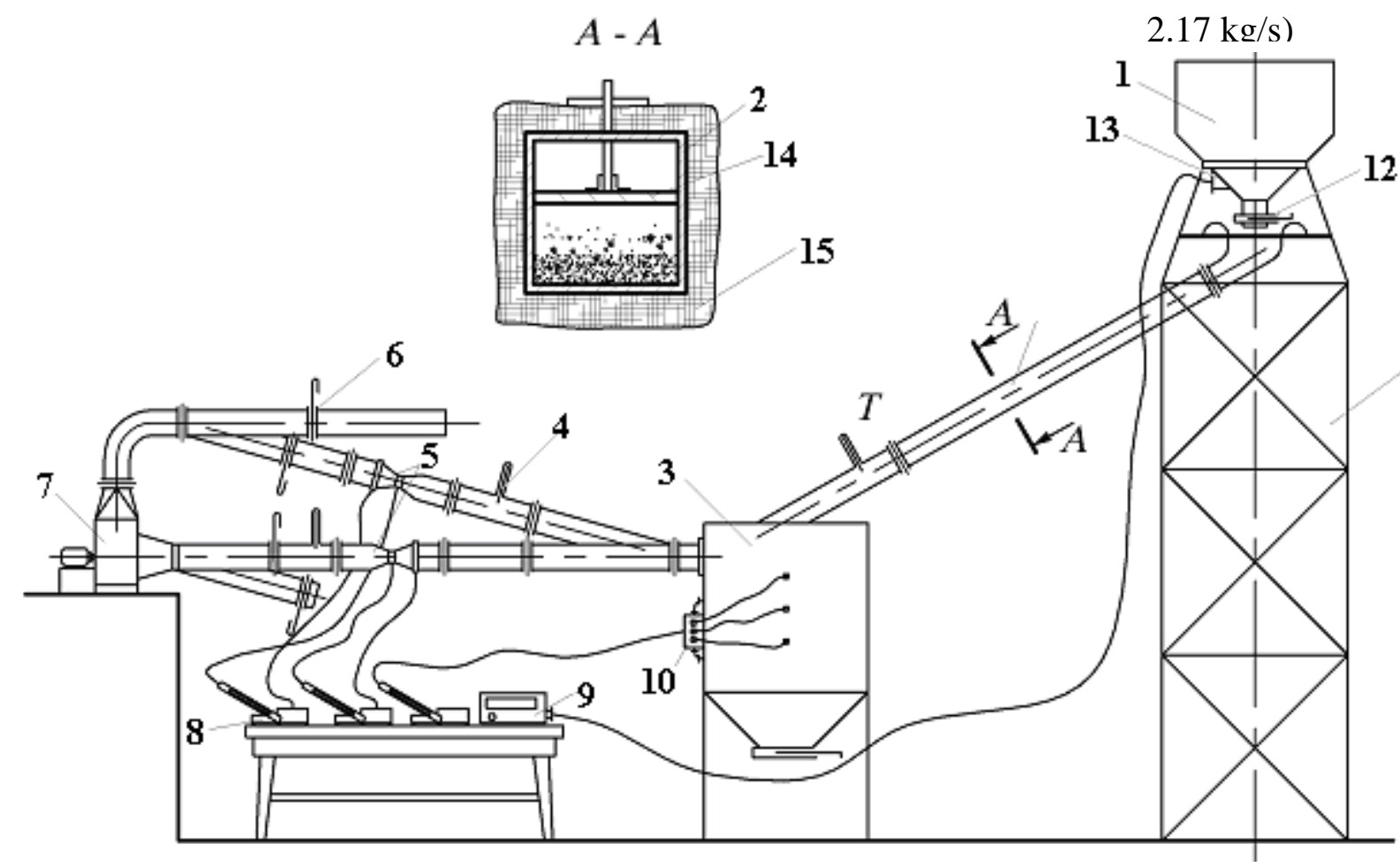

Figure 4: Diagram of the experimental arrangement for the study of injective properties of bulk materials: 1 upper bin; 2 - chute; 3 - lower bin; 4 - thermometer; 5 -Venturi tube; 6 - damper; 7 - fan; 8 - micropressure gauge; 9 - galvanometer; 10 - blending chamber; 11 - metal frame; 12 - diaphragm;13 - thermocouple; 14 chute upper wall; 15 - heat insulation layer

\section{CONCLUSIONS}

- The conducted studies are aimed at improving the theoretical basis for design of ventilated suction hoods of bulk material transfer groups, i.e. the most typical and common sources of dust emissions in reprocessing of dust-forming materials. The basic findings and resulting conclusions are as follows:

- It was theoretically demonstrated that at instant chuting of a heated bulk material an increase in the induction pressure is strongly related with a variation in the transferred material flow (54), and due to a considerable inertness of the intercomponent heat exchange temperature variations are far behind of variations in the transferred material quantity (Figure 2). A gradual variation in the flow rate of particles at the beginning and at the end of a material transfer is associated with excessive (peak) pressures against steadystate values (Figure 3). This is explained by the maximum induction head at a certain 
material flow rate $(67,68)$. There are no pressure surges if the material flow rate does not reach the necessary value.

\section{ACKNOWLEDGEMENTS}

The reported study was funded by RFBR according to the research project № 14-41-08005 ofi_m и and Russian President Scholarship СП-121.2016.1.

\section{REFERENCES}

[1] I.N. Logachev, K.I. Logachev, O.A. Averkova, Local Exhaust Ventilation: Aerodynamic Processes and Calculations of Dust Emissions. Boca Raton: CRC Press, 2015.

[2] I.N. Logachev, K.I. Logachev, Industrial Air Quality And Ventilation: Controlling Dust Emissions. Boca Raton, 2014.

[3] Ze Qin Liu. Air Entrainment in Free Falling Bulk Materials. Ph. D. Thesis. University of Wollngong, 2003. http:/ro.uow.edu.au/theses/1816.

[4] S. Mallick, P. W. Wypych. Evaluation of scaleup procedures using "system" approach for pneumatic conveying of powders. Particulate Sci. Technol. 28, No 1, 41-50, 2010.

[5] D. Hastie, P. Wypych. Researching conveyor belt trajectories. Engineers Australia, 81, 8, 2009.

[6] Li Xiaochuan, Wang Qili, Liu Qi, Hu Yafei. Developments in studies of air entrained by falling bulk materials. Powder Technology, 291, 159-169, 2016.

[7] Chenchen Fang, Jianliang Xu, Hui Zhao, Weifeng Li, Haifeng Liu. Experimental investigation on particle entrainment behaviors near a nozzle in gas-particle coaxial jets. Powder Technology, 286, 55-63, 2015.

[8] Koichiro Ogata, Katsuya Funatsu, Yuji Tomita. Experimental investigation of a free falling powder jet and the air entrainment// Powder Technology, 115, 90-95, 2001

[9] G.M. Fichtengolz. Calculus course. V.III. M: Fizmatgiz, 1963.

[10] O.D. Neykov, I.N. Logachev, R.N. Shumilov. Aspiration of vapor and dust mixtures in dedust-ing of process equipment. Kiev: Naukova dumka, 1974.

[11] G. Aramanovich, V.I. Levin. Equations of mathematical physics. M: Nauka, 1964.

[12] V.G. Shaptala, V.V. Shaptala, A.V. Gavrilenko, Numerical simulation of cement-air mixture in the pneumochamber pump. Bulletin of BSTU named after V.G. Shukhov, 2, 159-161, 2015.

[13] V.V. Shaptala, Modeling and calculation systems of cleaning dust emissions of cement production. Bulletin of BSTU named after V.G. Shukhov, 6, 205-209, 2015.

[14] V.G. Shaptala, V.V. Shaptala, The method of calculating efficiency of centrifugal collectors sticky dust in the production of building materials. Bulletin of BSTU named after V.G. Shukhov, 3, 58-62, 2014. 